\title{
The Darboux Transform Applied to Schrödinger Equations with a Position-Dependent Mass
}

\author{
G. Ovando ${ }^{1,2, *}$, J. Morales ${ }^{1}$, J.J. Peña ${ }^{1}$, G. Ares de Parga ${ }^{2}$ and J.L. López-Bonilla ${ }^{3}$ \\ ${ }^{1}$ Universidad Autónoma Metropolitana - Azcapotzalco, CBI - Area de Física Atómica Molecular Aplicada, Av. San \\ Pablo 180, Reynosa Azcapotzalco, 02200 México, D. F. \\ ${ }^{2}$ Dpto de Física, ESFM, Instituto Politécnico Nacional, Edif. 9, UP - ALM, Zacatenco, 07738 México, D.F. \\ ${ }^{3}$ SEPI-ESIME-Zacatenco, Instituto Politécnico Nacional, Edif. Z-4, 3er Piso, Col. Lindavista, 07738 México, D.F.
}

\begin{abstract}
Essentially, the Darboux proposition is based on the covariance properties of ordinary and partial differential equations with respect to a gauge transformation in the special case of second order differential equations of the SturmLiouville type. In this work, the one-dimensional Schrödinger equation with a position-dependent mass (SEPDM) is transformed into a Schrödinger-like equation with a position-independent mass (SLEPIM) for an effective potential which incorporates the spatially dependent mass. Therefore, taking advantage of the similarity between the SLEPIM and the Sturm-Liouville differential equation it is shown the application of the Darboux transform to the SEPDM problem.
\end{abstract}

Keywords: Darboux transform, Schrödinger equation, position-dependent mass.

\section{INTRODUCTION}

The one-dimensional Schrödinger equation with a position-dependent mass (SEPDM) occurs in the quantum chemistry study of microstructures such as the electronic properties of semiconductors, liquid crystals, quantum dots and non uniform materials in which the carrier effective mass depends on the position [1-4]. Consequently, the exactly solvable SEPDM has attracted considerable attention as demonstrated by already published methods on the subject such as the kinetic energy operator [5], Lie algebras [6,7], supersymmetry [8] and path integration [9] approaches. On the other hand, the Darboux transform (DT) [10] provides a very advisable way to construct new solutions of integrable equations by a purely algebraic algorithm. For this reason, the DT has been extensively used in quantum mechanics in the search of isospectral potentials for exactly solvable Schrödinger equations of constant mass (SLEPIM) [11-14], and nevertheless, only recently it has been applied to the SEPDM problem $[15,16]$. Thus, considering the aforesaid aspects, as well as the correspondence between the SturmLiouville (SL) equation and the SEPDM, this work has two objectives: a general approach to transform the SEPDM onto the SLEPIM in the search of exactly solvable SEPDM and the application of the DT to the SEPDM problem to find their corresponding isospectral potentials as given in the next two sections respectively.

\section{TRANSFORMATION OF THE SEPDM INTO A SLEPIM PROBLEM}

The point canonical transformation, first used by De et al. [17] in the mapping of shape invariant potentials, also

*Address correspondence to this author at the Universidad Autónoma Metropolitana - Azcapotzalco, CBI - Area de Física Atómica Molecular Aplicada, Av. San Pablo 180, Reynosa Azcapotzalco, 02200 México, D. F; E-mail: gaoz@correo.azc.uam.mx has been applied to the SEPDM [18]. However, of all the cases appeared in the literature, only particular positiondependent mass distributions have been studied, instead of obtaining all those distributions that fulfill exactly solvable SEPDM, in the assumed potential model. Thus, to overcome such difficulty, we have considered convenient to begin with two important definitions.

Definition 1. The SEPDM is a problem that looks for square integrable solutions $\psi_{\mathrm{n}}(\mathrm{x})$ on the interval $(-\infty, \infty)$, of the Sturm-Liouville equation

$$
-\frac{d}{d x}\left(\frac{1}{2 m(x)} \psi_{n}^{\prime}(x)\right)+V(x) \psi_{n}(x)=E_{n} \psi_{n}(x)
$$

where the prime denotes derivative with respect to the argument, $n$ is the number of zeros of the solutions, $V(x)$ is usually known as the potential function and $E_{n}$ are the corresponding eigenvalues.

Definition 2. Each integrable function $m(x) \geq 0$ defines a point canonical transformation $\mathrm{T}_{m}$ from the variable $x \in(-\infty$, $\infty$ ) onto new variable $u$ by the formula $u=g(x)=$ $\int^{x} \sqrt{2 m(t)} d t$. The inverse transformation, which is well defined, will be denoted by function $F(u)$, that is, $x=F(u)=$ $g^{-1}(u)$.

We are now in conditions for writing the equivalence between the SEPDM problem and the ordinary Schrödinger equation problem, i.e. the SLEPIM.

Theorem 1. Given a solution $\psi_{n}(x)$ of the SEPDM with potential $V(x)$ and mass $m(x)$ there exists a corresponding solution $\varphi_{n}(u)$ (in variable $u$ as defined by $\mathrm{T}_{m}$ ) of the SLEPIM

$-\varphi_{n}^{\prime \prime}(u)+V_{e f f}(u) \varphi_{n}(u)=E_{n} \varphi_{n}(u)$ 
where the effective potential $V_{\text {eff }}(u)$ is given in terms of the potential $V(F(u))$ and of the superpotential $W(u)$ by

$V_{\text {eff }}(u)=V(F(u))+W^{2}(u)+W^{\prime}(u)$,

where

$W(u)=\frac{d}{d u} \ln (2 m(F(u)))^{-\frac{1}{4}} ;$

the relation between the functions $\psi_{n}(x)$ and $\varphi_{n}(u)$ is a similarity transformation given by

$\psi_{n}(F(u))=\varphi_{n}(u) \exp \left[-\int^{u} W(t) d t\right]$

and the eigenvalues $E_{n}$ are the same for the SEPDM and the SLEPIM.

Proof. First we write the Eq. (1) in the form

$\frac{1}{2 m(x)} \psi_{n}^{\prime \prime}(x)+\left(\frac{1}{2 m(x)}\right)^{\prime} \psi_{n}^{\prime}(x)+\left(E_{n}-V(x)\right) \psi_{n}(x)=0$

and next we substitute the expressions

$\left(\frac{1}{2 m(x)}\right)^{\prime} \frac{d}{d x}=\frac{d \ln \left(\frac{1}{2 m(F(u))}\right)}{d u} \frac{d}{d u}$,

and

$\frac{1}{2 m(x)} \frac{d^{2}}{d x^{2}}=\frac{d^{2}}{d u^{2}}+\frac{d \ln \sqrt{(2 m(F(u))}}{d u} \frac{d}{d u}$,

to obtain

$\frac{d^{2}}{d u^{2}} \psi_{n}(F(u))+2 W(u) \frac{d}{d u} \psi_{n}(F(u))$

$+\left(E_{n}-V(F(u)) \psi_{n}(F(u))=0\right.$,

where $W(u)$ is defined by Eq. (4). Now we use Eq. (5) followed by Eq. (3) into the previous equation in order to get the Schrödinger equation given in Eq. (2).

Reciprocal theorem. Given a solution $\varphi_{n}(u)$ of the SLEPIM with effective potential $V_{\text {eff }}(u)$ and given the former potential $V(x)$ of a SEPDM problem, there exists a solution $\psi_{n}(x)$ in the variable $x$ of the SEPDM

$-\frac{d}{d x}\left(\frac{1}{2 m(x)} \psi_{n}^{\prime}(x)\right)+V(x) \psi_{n}(x)=E_{n} \psi_{n}(x)$,

with eigenfunctions

$\psi_{n}(x)=\varphi_{n}(g(x))[2 m(x)]^{\frac{1}{4}}$,

and mass distribution given by

$m(x)=\frac{1}{2} \exp \left[-4 \int^{g(x)} W(t) d t\right]$

being $W(u)$ solution of

$V(F(u))=V_{e f f}(u)-W^{2}(u)-W^{\prime}(u)$.
Proof: The reciprocal theorem follows a similar proof of theorem 1.

\section{THE DARBOUX TRANSFORM APPLIED TO THE SEPDM}

In order to know on the implications of the Darboux transform applied to the SEPDM problem, first we consider the Darboux transform applied to the SLEPIM problem.

According to the Darboux statement [10], we can define a Darboux transform from any given solution $\varphi_{p}(u)$ of the SLEPIM by using its logarithmic derivative which is $\sigma_{p}(u)=$ $\frac{d}{d u} \ln \varphi_{p}(u)$. The Darboux tranform is

$\varphi_{n}^{D}(u)=\left(\frac{d}{d u}-\sigma_{p}(u)\right) \varphi_{n}(u)$.

Darboux theorem: The function $\varphi_{n}^{D}(u)$ satisfies the SturmLiouville equation

$-\varphi_{n}^{D^{\prime \prime}}(u)+V_{\text {eff }}^{D}(u) \varphi_{n}^{D}(u)=E_{n} \varphi_{n}^{D}(u)$

with a new potential usually named the Darboux potential $V_{e f f}^{D}$

$V_{\text {eff }}^{D}=V_{\text {eff }}(u)-2 \sigma_{p}^{\prime}(u)$.

The proof of the Darboux theorem is standard and is given elsewhere [19].

With these elements, we are now in position to establish our main theorem:

Theorem 2. Suppose that $\psi_{n}(x)$ is solution of the SEPDM problem, $\varphi_{n}(u)$ is a solution of the SLEPIM problem, and both are related in the sense of Theorem 1 or reciprocal theorem. Then the function

$\psi_{n}^{D}(x)=\varphi_{n}^{D}(g(x))(2 m(x))^{1 / 4}$,

constructed from the Darboux transform $\varphi_{n}^{D}(u)$, is solution of the SEPDM problem given by

$-\frac{d}{d x}\left(\frac{1}{2 m(x)} \psi_{n}^{D^{\prime}}(x)\right)+V^{D}(x) \psi_{n}^{D}(x)=E_{n} \psi_{n}^{D}(x)$

where $V^{D}(x)$ is a new isospectral potential, named Darboux potential, which expression is

$V^{D}(x)=V(x)-\frac{2}{\sqrt{2 m(x)}} \frac{d}{d x}\left[\sigma_{p}(g(x))\right]$.

Proof. From the reciprocal theorem applied to Eq. (15), the Darboux potential for the SEPDM is

$V^{D}(F(u))=V_{e f f}^{D}(u)-W^{2}(u)-W^{\prime}(u)$,

using Eq. (16) is

$V^{D}(F(u))=V_{e f f}(u)-2 \sigma_{p}^{\prime}(u)-W^{2}(u)-W^{\prime}(u)$

and from Eq. (13) it is 


$$
V^{D}(F(u))=V(F(u))-2 \sigma_{p}^{\prime}(u)
$$

that matches with Eq. (19) after performing change of variable by means of the point canonical transformation $\mathrm{T}_{m}$.

\section{CONCLUDING REMARKS}

As can be appreciated, this work had a double purpose; firstly, to contribute to the study of exactly solvable SEPDM, and secondly, to find the isospectral potentials associated to the SEPDM problem. To the first purpose, we have proposed a gauge and a canonical point transformations, to convert the SEPDM into a SLEPIM problem; i.e. the method leads to the possibility of using the effective potentials involved in exactly solvable SLEPIM. To attain the second objective, the DT has been applied to the SEPDM problem by using like intermediary the SLEPIM. In both cases, the methods are general and can be straightforwardly applied to specific former potentials in order to find the corresponding mass distributions that guarantee the exact solvability of the SEPDM.

That is, our proposal is by far simpler than other approaches already published such as [15] where the position-dependent mass problem is focused by means of the Darboux transform and form-preserving transformations or as in [8] where the supersymmetric treatment of the SEPDM is given for a particular position-dependent mass and potential.

For example, to better appreciate the usefulness of our method in the search of exactly solvable SEPDM, it should be noticed that several situations can be identified and applied to the potential $V_{\text {eff }}(u)=V(F(u))+W^{2}(u)+W^{\prime}(u)$. Particularly, the simplest potential of the SEPDM is the null potential or free particle model in the $x$ space. That is, if the former potential $V(F(u))=V(x)=0$; then the effective potential is given by the Riccati equation

$V_{\text {eff }}(u)=W^{2}(u)+W^{\prime}(u)$,

from which many solvable effective potentials may be considered depending on the choice of the ansatz $W(u)$, that is related as well to the mass distribution. Therefore, the null potential model in the $x$ space for a certain $m(x)$ (SEPDM) is linked to an exactly solvable effective potential with constant mass in the $u$ space (SLEPIM) [20].

Another form to appreciate the scope of the Darboux transform applied to the SEPDM is to point out about the general relationship

$\sigma_{n}(u)^{2}+\sigma_{n}^{\prime}(u)+E_{n}=V(F(u))+W^{2}(u)+W^{\prime}(u)$,

which reduces to

$\sigma_{n}(u)^{2}+\sigma_{n}^{\prime}(u)+E_{n}=W^{2}(u)+W^{\prime}(u)$.

in the particular case of the free particle potential model. Consequently, if in addition $E_{0}=0$ one leads to $\sigma(u)=W(u)$ for which the potential $V^{D}(x)$ of the SEPDM problem becomes

$V^{D}(x)=-\frac{2}{\sqrt{2 m(x)}} \frac{d}{d x}[W(g(x))]$

that is equivalent to
$V^{D}(x)=\frac{1}{4} \frac{1}{(m(x))^{2}} \frac{\partial^{2} m(x)}{\partial x \partial x}-\frac{3}{8} \frac{1}{(m(x))^{3}} \frac{\partial m(x)^{2}}{\partial x}$

with eigenfunctions

$\varphi_{n}^{D}(u)=\left(\frac{d}{d u}-W(u)\right) \varphi_{n}(u)$.

In short, the proposals are general and can be used in the search of those exactly solvable SEPDM and their isospectral partners that fulfill specific former potential models and position-dependent mass distributions which in turn could be useful in the quantum mechanics treatment of outstanding applications in materials science.

\section{ACKNOWLEDGMENTS}

This work was partially supported by the projects UAMA-CBI-2232004 and CONACYT-32762-E, México. One of us (GO) want to acknowledge to the Instituto Politécnico Nacional for the hospitality in the realization of Ph.D. studies in applied mathematics and physics.

\section{REFERENCES}

[1] Geller MR, Kohn W. Quantum mechanics of electrons in crystals with graded composition. Phys Rev Lett 1993; 70(20): 3103-6.

[2] Serra L, Lipparini E. Spin response of unpolarized quantum dots. Europhys Lett 1997; 40(6): 667-72.

[3] Bastard G. Wave mechanics applied to heterostructures. Les Ulis: Les Éditions de Physique 1989.

[4] Barranco M, Gatica SM, Hernandez ES, Navarro J, Pi M. Structure and energetics of mixed ${ }^{4} \mathrm{He}-{ }^{3} \mathrm{He}$ drops. Phys Rev B 1997; 56(14): 8997-9003.

[5] Bagchi B, Gorain P, Quesne C, Roychoudhury R. A general scheme for the effective-mass Schrödinger equation and the generation of the associated potentials. Mod Phys Lett A 2004; 19(37): 2765-75.

[6] Roy B. Lie algebraic approach to singular oscillator with a position-dependent mass. Europhys Lett 2005; 72(1): 1-6.

[7] Dong SH, Peña JJ, Pacheco-García C, García-Ravelo J. Algebraic approach to the position-dependent mass Schrödinger equation for a singular oscillator. Mod Phys Lett A 2007; 22(14): 1039-45.

[8] Gönul B, Gönul B, Tutcu D, Özer O. Supersymmetric approach to exactly solvable systems with position-dependent effective masses. Mod Phys Lett A 2002; 17(31): 2057-66.

[9] Mandal BP. Path integral solution of noncentral potential. Int J Mod Phys A 2000; 15(8): 1225-34.

[10] Darboux MG. Sur une proposition relative aux équations linéaires. Comptes Rendus Acad Sci 1882; 94: 1456-59.

[11] Morales J, Peña JJ, López-Bonilla JL. Generalization of the Darboux transform. J Math Phys 2001; 42: 966-75.

[12] Peña JJ, Ovando G, Morales-Guzmán D, Morales J. Solvable quartic potentials and their isospectral partners. Int J Quant Chem 2001; 85: 244-50.

[13] Morales J, Peña JJ, Rubio-Ponce A. New Isospectral generalized potentials. Theor Chem Acc 2003; 110: 403-9.

[14] Rubio-Ponce A, Peña JJ, Morales J. One-parameter isospectral solutions for the Fokker-Planck equation. Phys A 2004; 339: 28595 .

[15] Schulze-Halberg A. Effective mass hamiltonians with linear terms in the momentum: Darboux transformations and form-preserving transformations. Int J Mod Phys A 2007; 22: 1735-69.

[16] Schulze-Halberg A. Darboux transformations for effective mass Schrödinger equations with energy-dependent potentials. Int J Mod Phys A 2008; 23: 537-46.

[17] De R, Dutt R, Sukhatme U. Mapping of shape invariant potentials under point canonical transformations. J Phys A: Math Gen 1992; 25: L843-50.

[18] Jiang L, Yi LZ, Jia CS. Exact solutions of the Schrödinger equation with position-dependent mass for some Hermitian and nonHermitian potentials. Phys Lett A 2005; 345: 279-86. 
[19] Matveev VB, Salle MA. Darboux transformations and solitons. Berlin: Springer-Verlag 1991.
[20] Peña JJ, Ovando G, Morales J, García-Ravelo J, Pacheco-García C. Exactly solvable Schrödinger equations with a position-dependent mass: null potential. Int J Quant Chem 2007; 107: 3039-45. et al.; Licensee Bentham Open.

This is an open access article licensed under the terms of the Creative Commons Attribution Non-Commercial License (http://creativecommons.org/licenses/bync/3.0/), which permits unrestricted, non-commercial use, distribution and reproduction in any medium, provided the work is properly cited. 